\title{
L'ipertensione arteriosa nel follow-up del trapianto renale
}

\author{
D. Angelini, M. Parrini, A. Rosati
}

U.O. Nefrologia, Ospedale Campo di Marte, Lucca

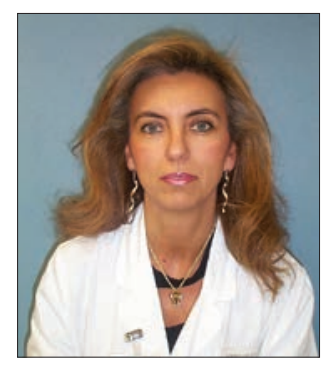

Daniela Angelini
Lipertensione arteriosa è una complicanza frequente nel followup del trapianto renale. Si manifesta in oltre il $75 \%$ dei trapianti renali ed è associata a una maggior percentuale di morte prematura o di perdita del rene. Le cause di ipertensione nel trapiantato sono molteplici ma la prevalenza varia significativamente con il tipo di terapia immunosoppressiva ed è infatti passata dal 30-40\%, nel periodo Pre-Ciclosporina (CyA), al 75-90\% dopo l'introduzione degli inibitori della calcineurina (CNI) (1). Studi osservazionali hanno dimostrato un incremento di circa 1'1-2\% del rischio di eventi cardiovascolari (CV) fatali e non fatali per ogni incremento di $1 \mathrm{mmHg}$ di pressione sistolica $(2,3)$. Uno studio eseguito su 29.751 pazienti seguiti per oltre 7 anni ha dimostrato che l'ipertensione non controllata si associa a un graduale peggioramento della funzione renale valutata a 1,3 e 5 anni dal trapianto (6). Non ci sono trials randomizzati che abbiano definito i target pressori nel paziente trapiantato, ma, sulla base degli studi condotti sulla popolazione generale con o senza compromissione della funzione renale, le linee guida KDOQI raccomandano, nei pazienti con trapianto renale, un target di $130 / 80 \mathrm{mmHg}$ in assenza di proteinuria e $120 / 75 \mathrm{mmHg}$ se coesiste proteinuria (4). Un adeguato trattamento anti-ipertensivo nei portatori di trapianto renale è quindi di fondamentale importanza non solo per la sopravvivenza dell'organo trapiantato ma anche per contenere l'aumento del rischio cardiovascolare e quindi della morbidità e mortalità CV che rappresenta il $30 \% \mathrm{e}$ il $75 \%$ dei decessi post-trapianto rispettivamente precoci e tardivi $(4,5)$. L'ipertensione post-trapianto però è spesso difficile da controllare e possono essere necessari più farmaci. Un attento giudizio clinico e le necessarie conoscenze di farmacodinamica e farmacocinetica sono di fondamentale importanza quando si inizia una terapia.

\section{Cause di ipertensione post-trapianto (Tab. I)}

I fattori causali sono molteplici ma i più frequentemente coinvolti sono l'ipertensione pre-trapianto, la CAN e la terapia con inibitori della calcineurina (CNI). O1tre il 50\% dei trapiantati presenta infatti ipertensione pre-trapianto sia come causa di IRC che come conseguenza. Tali pazienti presentano alterazioni funzionali e strutturali delle pareti arteriose con aumento della rigidità delle grandi arterie ed evidenza di calcificazioni vascolari. Queste alterazioni patologiche persistono dopo il trapianto e contribuiscono all'ipertensione arteriosa sia nei trapiantati da donatore vivente che da cadavere (6). A tale riguardo Delahousse et al (7) hanno dimostrato una correlazione diretta tra la PA misurata a 3 mesi e a 12 mesi dal trapianto renale e la rigidità aortica. Non è però da sottovalutare l'ipertensione legata al donatore: i riceventi di trapianto renale da donatore cadavere iperteso o con storia familiare di ipertensione arteriosa, non in terapia con CNI, presentano un aumento del rischio di sviluppare ipertensione arteriosa (8). Uno studio retrospettivo di Ducloux et al (9) sul ruolo del donatore e del ricevente nello sviluppo di ipertensione, eseguito su 321 riceventi con trapianto funzionante a 1 anno, ha dimostrato come il BMI e l'età del donatore siano correlate all'ipertensione. Fra i pazienti in terapia con CNI quelli trattati con CyA erano più predisposti a sviluppare ipertensione rispetto a quelli in terapia con Tacrolimus (10). Kasiske et al (3) hanno dimostrato in uno studio su 1600 pazienti seguiti per oltre 20 anni come possano essere considerati fattori predisponenti allipertensione post-trapianto: il sesso maschile, l'età del ricevente, il BMI, il rigetto acuto, bassi livelli di emoglobina, la terapia con CyA e alte dosi di prednisone.

Per quanto riguarda i meccanismi fisiopatologici dell'ipertensione indotta dai $\mathrm{CNI}$, sebbene non siano ancora 
TABELLA I - CAUSE DI IPERTENSIONE ARTERIOSA POSTTRAPIANTO

\section{Correlate al ricevente}

- Ipertensione arteriosa preesistente con ipertrofia ventricolare sinistra

- Body mass index

- Malattia renale primitiva (reni nativi)

Correlate al donatore

- $\quad$ Età e sesso femminile

- Ipertensione arteriosa

Correlate al trapianto

- Ischemia prolungata

- Ritardata ripresa della funzione dell'organo

Terapia immunosoppressiva

- Inibitori della calcineurina (CyA e TAC)

- $\quad$ Corticosteroidi

Stenosi dell'arteria renale del rene trapiantato

Ostruzione ureterale, linfocele

Disfunzione del trapianto renale (CAN, GN)

Modificata da

Castillo Lugo J.A e Marini P.V. Hypertension in Kidney Transplantation. Sem in Nephrol 2005; 25: 252-60.

completamente conosciuti, comprendono:

1. Un aumento delle resistenze periferiche per diffusa vasocostrizione arteriosa (11) che a sua volta è legato a :

a) attivazione da parte dei CNI del sistema renina angiotensina (RAS) per effetto diretto sulle cellule iuxtaglomerulari e indirettamente attraverso una vasocostrizione renale (6);

b) aumento legato ai CNI dell'effetto vasocostrittore dell'Angiotensina II (12). Si determina uno squilibrio fra fattori vasocostrittori e vasodilatanti con riduzione di molecole vasodilatanti come prostaglandine e ossido nitrico e un aumento di sostanze ad azione vasocostrittrice come PDGF ed endotelina (8);

c) intensa vasocostrizione dell'arteriola afferente renale per stimolazione del SNS e sovraregolazione locale del sistema renina-angiotensina-aldosterone con possibile riduzione del filtrato glomerulare e conseguente aumento del riassorbimento tubulare di sodio (6).

2. Ł̀ ormai dimostrato che la CyA stimola l'espressione per il recettore dell'angiotensina nelle cellule muscolari lisce, determinando una maggiore vasocostrizione, uno stimolo all'aterogenesi per proliferazione del- le cellule muscolari lisce stesse e aumento degli indici di flogosi (13). Il Tacrolimus probabilmente agisce con gli stessi meccanismi ma alcuni studi di conversione da ciclosporina a Tacrolimus hanno dimostrato la riduzione significativa della pressione arteriosa che può anche essere interpretata come espressione di un miglioramento della funzione renale (8).

Steroidi: Anche gli steroidi giocano un ruolo importante nella patogenesi dell'ipertensione post-trapianto, legata in gran parte alla ritenzione idrosalina. Studi più recenti hanno suggerito che l'ipertensione da steroidi potrebbe essere il risultato dell'attivazione mediata dai recettori dei glucocorticoidi delle cellule muscolari lisce vasali (14). Studi di sospensione degli steroidi hanno dimostrato un miglior controllo della pressione arteriosa, una riduzione del rischio $\mathrm{Cv}$ con riduzione della percentuale di rigetti e di perdita del trapianto (15).

La stenosi dell'arteria renale del trapianto (TRAS) è responsabile dall'1 al 7\% delle ipertensioni post-trapianto. Il meccanismo ipertensivo è legato all'attivazione del SRAA con conseguente vasocostrizione sistemica e aumento del riassorbimento di acqua e sodio. Clinicamente questo tipo di ipertensione può essere refrattario alla terapia medica e può associarsi a un peggioramento della funzione renale ed edemi periferici. Per la diagnosi è fondamentale l'ecocolordoppler e ancora più specifiche sono l'AngioTC spirale e l'Angio Risonanza Magnetica. La terapia è l' angioplastica percutanea con o senza stenting (16).

Ma quanto la presenza dei reni nativi contribuisce all'ipertensione post-trapianto? In uno studio di Huysmans et al (17) la prevalenza di ipertensione era più elevata nei pazienti con reni nativi rispetto ai pazienti senza, nella percentuale rispettivamente del 57,8\% e 23,9\%. L'esatto ruolo dei reni nativi sul persistere di ipertensione nel post- trapianto è comunque ancora da chiarire. Numerosi studi hanno dimostrato che la nefrectomia bilaterale dei reni nativi in pazienti con ipertensione resistente, eseguita sia prima che dopo il trapianto, determina un miglior controllo pressorio in molti pazienti ma non in tutti (18) anche se d'altra parte la nefrectomia è sempre meno utilizzata grazie anche al miglioramento delle terapie farmacologiche.

\section{Terapia dell'ipertensione post-trapianto}

\section{La terapia non farmacologica}

Le modificazioni dello stile di vita dovrebbero essere incoraggiate in tutti i pazienti con pre-ipertensione $\mathrm{e}$ 
ipertensione come primo step terapeutico e quindi anche nell'ipertensione post-trapianto. Non esistono però ancora studi controllati nei pazienti con trapianto renale anche se l'uso di una dieta adeguata (Dietary Approaches to Stop Hypertension eating plan, DASH), la riduzione del peso, la cessazione del fumo, l'attività fisica costante sono raccomandabili in tutti i pazienti trapiantati. Le modificazioni dietetiche sono particolarmente importanti in quanto una dieta ricca di sodio non solo può contribuire all'incremento pressorio ma può anche ridurre l'effetto anti-ipertensivo di alcune classi di farmaci (19). L'ingestione di elevate quantità di frutta e vegetali e una dieta ricca di prodotti a basso contenuto di grassi (dieta DASH) ha dimostrato in uno studio pubblicato negli ultimi mesi una drammatica riduzione della PA rispetto ai pazienti a dieta standard (20).

\section{Gli Immunosoppressori}

Il tipo e il dosaggio dei farmaci immunosoppressori giocano un ruolo importante nel controllo dell'ipertensione post-trapianto. Benché nella scelta non si tenga generalmente conto degli effetti sulla pressione arteriosa è però evidente come variazioni del regime immunosoppressivo possano determinare un miglioramento del controllo pressorio (6). I protocolli terapeutici privi dello steroide stanno progressivamente guadagnando popolarità e sono passati dal 5\% nel 2000 al 23\% nel 2004 al 26\% nel 2007 (6). I protocolli che prevedono la riduzione o la sospensione dello steroide in numerosi studi si associano a un miglioramento del controllo pressorio nel post-trapianto sia nella popolazione bianca che Afro Americana, sia negli adulti che nella popolazione pediatrica (21). Anche se non tutti gli studi concludono univocamente come il recente studio randomizzato, doppio cieco di Woodle et al (22) su 386 trapiantati randomizzati in pazienti a sospensione precoce dello steroide vs mantenimento dello steroide (5 mg a sei mesi): le PA sistolica e diastolica non hanno dimostrato variazioni significative nei due gruppi. Per quanto riguarda gli effetti dei CNI è noto che sia la CyA che il Tacrolimus determinano vasocostrizione sistemica ma il Tacrolimus ha un effetto meno severo e più ritardato sull'incremento delle resistenze periferiche e sulla pressione arteriosa rispetto alla Ciclosporina; la conversione da CyA a Tacrolimus si associa a un modesto ma significativo miglioramento del controllo pressorio (10).

\section{Terapia farmacologica}

Sebbene le linee guida non sostengano una classe preferita di farmaci anti-ipertensivi nel trapianto renale, i
Calcio Antagonisti possono essere considerati farmaci di prima scelta fin dalle prime fasi del trapianto. Hanno un effetto dilatante favorevole sull'arteriola afferente ed è stata dimostrata la loro azione di contrasto sull'effetto vasocostrittore dei CNI (1). Come dimostrato da Cross et al in una metanalisi condotta su 17 studi controllati e randomizzati che coinvolgevano 1255 trapiantati, l'uso dei Calcio Antagonisti riduceva il rischio di perdita del rene del 25\% e migliorava il filtrato glomerulare e la creatininemia. In particolare i diidropiridinici migliorerebbero la funzione renale se confrontati con gli ACE-I (1). Anche i non diidropiridinici possono essere utilizzati ed è stato dimostrato il loro effetto sinergico antiproteinurico quando utilizzati insieme agli ACE-I. Questi interagiscono anche con CYP3A4 e P-Glicoproteine determinando un aumento della concentrazione di CNI per cui $\square$ quando utilizzati $\square$ è necessario monitorare il dosaggio degli immunosoppressori, data l'estrema variabilità da paziente a paziente.

Gli ACE-I sono spesso i farmaci di seconda scelta nell'ipertensione post-trapianto. Numerosi studi hanno dimostrato che a 6-12 settimane dal trapianto sono sicuri ed efficaci nella riduzione della PA (1). Hanno il vantaggio di favorire la riduzione del post-carico, nonché diminuire il tasso di escrezione di proteine nei pazienti con proteinuria (23). Sia gli ACE-I che gli ARBs sono agenti efficaci nel trattamento dell'eritrocitosi post-trapianto e sono pertanto considerati farmaci di scelta nel trattamento dei pazienti ipertesi con tale coesistente patologia (25). Nell'immediato periodo post-trapianto molti clinici sono esitanti ad iniziare de novo una terapia con ACE o ARBs in quanto l'incremento della creatininemia che possono determinare può mascherare un rigetto; ma i pazienti che erano già stabili con tale terapia prima del trapianto dovrebbero reiniziare questa terapia nel post-trapianto se indicata (1). Dobbiamo inoltre sempre tenere presente che questi farmaci hanno un effetto importante sulla riduzione del rischio cardiovascolare determinando una riduzione dell'ipertrofia ventricolare sinistra e migliorando la rigidità arteriolare (1). Nella metanalisi di Cross et al sono stati presi in esame 7 trials controllati di confronto fra ACE-I e Ca-antagonisti: il GFR risultava significativamente ridotto, così come la creatininemia aumentava nei pazienti in ACE-I rispetto a quelli in terapia con Ca-antagonisti, compresi i pazienti con proteinuria (24).

Quando si usano questi farmaci è consigliato comunque partire con basse dosi e monitorare strettamente la funzione renale, la potassiemia e l'emoglobina. Può essere indicata anche un'ecodoppler per escludere la presenza di stenosi dell'arteria renale, prima di iniziare la terapia (24). Nei pazienti trapiantati ipertesi con sovraccarico di volume, che presentano edemi periferici e aumento di peso 
è indicata l'aggiunta di diuretici dell'ansa. Essi agiscono sinergicamente specialmente con gli ACE-I nella riduzione dei valori pressori. Grazie alla loro rapida azione spesso, in questi casi, i diuretici dell'ansa sono farmaci di scelta specialmente se usati per via parenterale.

Lo Spironolattone è un antagonista dell'Aldosterone che è efficace nel controllo dell'ipertensione sia nella popolazione generale che nell'IRC anche nei pazienti che non presentano agli esami di laboratorio evidenza di incremento di Aldosterone. Ha inoltre proprietà renoprotettive come ampiamente dimostrato nella nefropatia diabetica (6). Nel trapianto renale induce la natriuresi e migliora la PA nell'uomo e migliora la vasculopatia da trapianto nei modelli animali. L'aggiunta di Spironolattone limita la fibrosi tubulo interstiziale indotta dai CNI e previene l'ulteriore progressione del danno renale nei ratti con nefropatia da CNI (25).

I Beta-bloccanti sono spesso utilizzati nella terapia antiipertensiva nel post-trapianto, soprattutto nei pazienti che già ne facevano uso precedentemente; da tenere presente che tali farmaci favoriscono l'insorgenza di bradicardia, disfunzione sessuale, iperlipemia, complicanze che sono spesso già presenti nei pazienti trapiantati. I Beta-bloccanti di terza generazione cha combinano alfa e beta blocco come il Carvedilolo hanno una maggiore potenza anti-ipertensiva e un limitato o benefico effetto sul profilo lipidico (26). Il loro uso è soprattutto raccomandato se esistono indicazioni specifiche per tale terapia come angina, aritmie, insufficienza cardiaca e nei pazienti con precedenti di infarto miocardico acuto nei quali hanno dimostrato un effetto cardioprotettivo con miglioramento della sopravvivenza a lungo termine (6). Gli Alfa1 antagonisti sono da riservare come terapia aggiuntiva nei pazienti con ipertensione più difficilmente controllabile $o$ in pazienti con concomitante ipertrofia prostatica benigna sintomatica. Essi dovrebbero essere somministrati al momento di coricarsi, al dosaggio più basso di partenza per i pazienti näive, a causa dell'elevato rischio di ipotensione posturale nella fase iniziale (6). Infine pazienti con ipertensione resistente possono avere necessità di farmaci ad azione centrale come la Clonidina, sia in compresse che per via transdermica.

\section{Conclusioni}

L'ipertensione rappresenta un problema importante nella gestione del paziente trapiantato e contribuisce a ridurre la sopravvivenza non solo del trapianto ma anche del paziente, determinando un aumento degli eventi fatali e non fatali cardiovascolari.

Il trattamento dell'ipertensione post-trapianto richiede un approccio multifattoriale con l'utilizzo simultaneo della terapia farmacologica e non farmacologica, tenendo conto delle spesso numerose comorbidità $\mathrm{e}$ delle interazioni con i farmaci immunosoppressori.

Le evidenze attuali non favoriscono una classe precisa di farmaci anche se i Ca-antagonisti ben si adattano ad essere il farmaco di prima scelta.

Le modifiche dei farmaci immunosoppressori, in particolare la riduzione o la sospensione di CNI, possono avere effetti positivi sul controllo della pressione arteriosa nel post-trapianto, ma con un aumento del rischio di rigetto.

Poiché la sopravvivenza del trapianto a lungo termine è strettamente legata alla riduzione della morbilità $\mathrm{e}$ mortalità $\mathrm{CV}$, è necessario uno stretto controllo della pressione arteriosa ed è auspicabile che studi futuri valutino nuovi farmaci immunosoppressori con minor impatto sulla pressione arteriosa.

\author{
Indirizzo degli Autori: \\ Daniela Angelini, MD \\ U.O di Nefrologia \\ Ospedale Campo di Marte \\ Via dell'Ospedale \\ 55100 Lucca \\ d.angelini@usl2.toscana.it
}

\section{Bibliografia}

1. Dunn BL, Teusink AC, Taber DJ, et al. Management of hypertension in renal transplant patients: a comprehensive review of nonpharmacologic and pharmacologic treatment strategies. The Annals of Pharmacol 2010; 44: 1259-70.
2. Kasiske BL, Anjum S, Shah R, et al. Hypertension after kidney transplantation. Am J Kidney Dis 2004; 43 (6): 1071-81.

3. Campistol JM, Romero R, Paul J, Gutierrez-Dolmau A. Epidemiology of arterial hypertension in renal transplant patients: changes over the last decade. Nephrol Dial 
Transplant 2004; 19 (Suppl 3); S62-6.

4. Ojo A. Cardiovascular complications after renal transplantation and their prevention. Transplantation 2006; 82: 603-11.

5. Hani M Wadei, Stephen C Textor. Hypertension in the kidney transplant recipient. Transplantation reviews 2010; 24: 105-20.

6. Delahousse M, Chaignon M, Mesnard 1, et al. Aortic stiffness of kidney transplant recipients correlates with donor age. J Am Soc Nephrol 2008; 19: 798-805.

7. Scolari MP, La Manna G, Cianciolo G, et al. I fattori di progressione della malattia cardiovascolare dopo trapianto renale. G Ital Nefrol 2009; 26(Suppl 546): S30-43.

8. Duclouxe D, Motte G, Kribs M, et al. Hypertension in renal transplantation: Donor and recipient risk factors. Clin Nephrol 2002; 57: 409-13.

9. Margreiter R. For the European Tacrolimus vs Cyclosporin Microemulsion Renal Transplantation Study Group. Efficacy and safety of tacrolimus compared with cyclosporine microemulsion in renal transplantation. A randomized multicentre study. Lancet 2002; 359: 741-6.

10. Textor SC, Canzanello VJ, Taler SJ, et al. Cyclosporine induced hypertension after transplantation. Mayo Clinic Proc 1994; 69: 1182-93.

11. Lassila M. Interaction of cyclosporine $A$ and the reninangiotensin system; new perspectives. Curr Drug Metab 2002; 3: 61-71.

12. Koonans HA, Ligtenberg G. Mechanisms and consequences of arterial hypertension after renal transplantation. Transplantation 2001; 72 (Suppl): S9-12.

13. Goodwin JE, Zhang J, Geller DS. A critical role for vascular smooth muscle in acute glucocorticoid induced hypertension. J Am Soc Nephrol 2008; 19: 1291-9.

14. Oberbauer R, Kreis H, Johnson RW, et al. Long term improvement in renal function with sirolimus after early ciclosporine withdrawal in renal transplant recipients: 2 years results of the rapamune Manintenance Regimen Study. Transplantation 2003; 76: 364-70.

15. Guzman-Rodriguez JH,Plata-Munoz JJ, Mancilla E, et al. Renal artery stenosis after transplantation treatment using percutaneous transluminal angioplasty and placement of an expandable metal luminal prosthesis. Rev Invest
Clin 2003; 55: 297-304.

16. Huysmans FT, Hoitsma AJ, Koene RA. Factors determining the prevalence of hypertension after renal transplantation. Nephrol Dial Transplant 1987; 2: 34-8.

17. Sanieevan KV, Bhat HS, et al. Laparoscopic simultaneous bilateral pretransplant nephrectomy for uncontrolled hypertension. Transplant Proc 2004; 36: 2011-12.

18. Calhoum DA, Jones D, Textor S, et al. Resistant hypertension: diagnosis, evaluation and treatment: a scientific statement from tha American Heart Association Professional Education Committee of the Council for High Blood Pressure Research. Hypertension 2008; 51: 1403-19.

19. US Department of Health and Human Services. Facts about the DASH eating plan. http://www.nblbi.nih.gov/ health/public/heart/hbp/dash/new_dash.pdf (accessed 2010 Jan 15)

20. Hocker B, Weber LT, Feneberg R, et al. Prospective, randomized trial on late steroid withdrawal in pediatric renal transplant recipients under cyclosporine microemulsion and mycophenolate mofetil. Transplantation 2009; 87: 934-41.

21. Woodle ESMD, First MRMD, Pirsh JMD, et al. A prospective, randomized double-blind, placebo-controlled multicenter trial comparing early (7 day) corticosteroid cessation versus longterm, low-dose corticosteroid therapy. Ann Surg 2008; 248: 564-77.

22. Cruzado JM, Rico J, Gringo JM. The renin angiotensin system blockade in kidney transplantation: pros and cons. Transplant Int 2008; 21: 304-13.

23. Cross NB, Webster AC, Masson P, O'Connell PJ, Craig JC. Antihypertensives for kidney Transplant recipients: Systematic review and meta-analysis of randomized controlled trials. Transplantation 2009; 88: 7-18.

24. Waanders F, Rientra H, Boer MW, et al. Spironolactone ameliorates transplant vasculopathy in real Chronic transplant dysfunction in rats. Am J Phisiol Renal Phisiol 2009; 296: F 1072-9.

25. Bell DS, Bakris GL, McGill JB. Comparison of carvedilol and methoprolol or serum lipid concentration in diabetic hypertensive patients. Diabetes Obes Metab 2009; 11: 234-8. 